\title{
Photonic Bandgap Microcavity Devices
}

\author{
A. Scherer, M. Loncar, T. Yoshie, K. Okamoto \\ Caltech, Pasadena, CA 91125
}

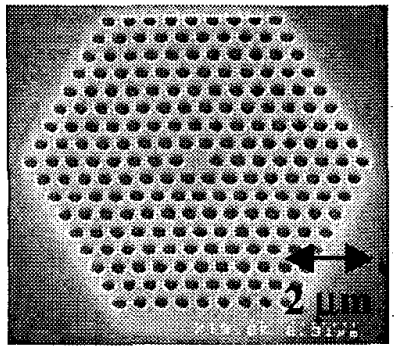

Figure 1. Photonic crystal nanocavity laser structure fabricated in InGaAsP

When combined with high index contrast slabs in which light can be efficiently guided, microfabricated two-dimensional photonic bandgap mirrors provide us with the geometries needed to confine and concentrate light into extremely small volumes and to obtain very high field intensities. Fabrication of optical structures has now evolved to a precision which allows us to control light within such etched nanostructures. Sub-wavelength nano-optic cavities can be used for efficient and flexible control over both emission wavelength and frequency, and nanofabricated optical waveguides can be used for efficient coupling of light between devices. The reduction of the size of optical components leads to their integration in large numbers and the possibility to combine different functionalities on a single chip, much in the same way as electronic components have been integrated for improved functionality in microchips. The past rapid emergence of optical microcavity devices, such as Vertical Cavity Surface Emitting Lasers (VCSELs) can be largely attributed to the high precision over the layer thickness control available during semiconductor crystal growth. High reflectivity mirrors can thus be grown with sub-nanometer accuracy to define high-Q cavities in the vertical dimension. Recently, it has become possible to microfabricate high reflectivity mirrors by creating two- and three-dimensional periodic structures. These periodic "photonic crystals" can be designed within materials systems with high refractive index contrast. This opportunity allows photonic bandgaps materials to be defined, with frequency ranges within which the propagation of electromagnetic waves is forbidden irrespective of the propagation direction in space to be defined. When combined with high index contrast slabs in which light can be efficiently guided, manufacturable two-dimensional photonic bandgap devices provide us with the geometries needed to confine and concentrate light into extremely small volumes and to obtain very high field intensities. Here we describe the design, the fabrication and the characterization of functional optical devices, such as lasers, modulators, add/drop filters, polarizers and detectors based on photonic crystals.

Figure 2.
Modeling
results
showing a
dipole mode in
a photonic
crystal cavity.
3-D finite
difference time
domain model

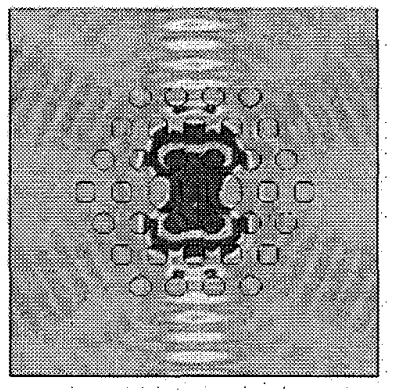

$2-2.00000$

We will show that the design and fabrication of optical structures has evolved to a precision which allows us to control light emission from etched nanostructures. For example, sub-wavelength nano-optic cavities can be used for efficient and flexible control over both emission wavelength and frequency. Similarly, nanofabricated optical waveguides can be used for efficient coupling of light between devices. This new capability enables the reduction of the size of optical components and leads to their integration in large numbers, much in the same way as electronic components have been 
integrated for improved functionality to form microchips. As high-Q optical and electronic cavity sizes approach a cubic half-wavelength the spatial and spectral densities (both electronic and optical) increase to a point where strong light-matter coupling becomes possible. We have developed new optical cavities with $Q$ values above 20,000 , and mode volumes as small as two to three cubic half wavelengths. With new designs, we have demonstrated optically pumped photonic crystal lasers with low threshold powers lasers, with threshold powers below $0.1 \mathrm{~mW}$. We have also incorporated self-assembled quantum dot emitters into our photonic crystal cavities, and defined quantum dot photonic crystal lasers. To verify our modeling predictions, we have also used near field scanning optical microscopy to locally probe the optical fields in our photonic crystal nanocavities. All of these efforts have been aimed at developing the discrete components which are expected to be used in future nanophotonic systems. Finally, we have defined photonic crystal laser sensors which can be used for the analysis of femtoliter volumes of analyte. 